\title{
Anomalous Hall Effect in Geometrically Frustrated Magnets
}

\author{
D. Boldrin and A. S. Wills \\ Department of Chemistry, University College London, 20 Gordon Street, London WC1H 0AJ, UK \\ Correspondence should be addressed to A. S. Wills, a.s.wills@ucl.ac.uk
}

Received 15 October 2011; Revised 21 December 2011; Accepted 22 December 2011

Academic Editor: Charles Rosenblatt

Copyright ( $) 2012$ D. Boldrin and A. S. Wills. This is an open access article distributed under the Creative Commons Attribution License, which permits unrestricted use, distribution, and reproduction in any medium, provided the original work is properly cited.

\begin{abstract}
Geometrically frustrated conducting magnets display extraordinarily large anomalous Hall effects (AHEs) that could be used to realise materials required for the emerging field of spintronics. While the intrinsic Berry phase developed in collinear ferromagnets is well explained through the effects of spin-orbit interactions within the Karplus and Luttinger model, its origins in frustrated magnets are not. The direct space mechanism based on spin chirality that was originally applied to the pyrochlore $\mathrm{Nd}_{2} \mathrm{Mo}_{2} \mathrm{O}_{7}$ appears unsatisfactory. Recently, an orbital description based on the Aharonov-Bohm effect has been proposed and applied to both the ferromagnetic pyrochlores $\mathrm{Nd}_{2} \mathrm{Mo}_{2} \mathrm{O}_{7}$ and $\mathrm{Pr}_{2} \mathrm{Ir}_{2} \mathrm{O}_{7}$; the first of which features long-ranged magnetic order while the latter is a chiral spin liquid. Two further examples of geometrically frustrated conducting magnets are presented in this paper-the kagome-like $\mathrm{Fe}_{3} \mathrm{Sn}_{2}$ and the triangular $\mathrm{PdCrO}_{2}$. These possess very different electronic structures to the 3-dimensional heavy-metal pyrochlores and provide new opportunities to explore the different origins of the AHE. This paper summarises the experimental findings in these materials in an attempt to unite the conflicting theoretical arguments.
\end{abstract}

\section{Introduction}

As the shrinking of components in integrated circuits characterised by Moore's law reaches its physical limit, the search is on for new properties and materials that can be used to carry, process, and store information. One of the key areas for research that could allow these improvements to continue is the field of magnetism where information is encoded in electronic spin rather than charge [1]. Current technologies have already benefited from some of this potential with the improvements in hard disk capacity being based on the developments of giant magneto-resistance (GMR) devices and in the synthesis of highly anisotropic magnetic nanoparticles that store the data. Magnetic random access memory (MRAM) based on spin-dependent tunneling is also becoming more affordable and shows advantages in power consumption and stability over conventional silicon-based electronics [2].

Developing the required suite of spin-dependent electronic, spintronic devices, requires the discovery of materials that display strong spin-dependent electronic properties at room temperature. Following on from initial work on the ferromagnetic pyrochlore $\mathrm{Nd}_{2} \mathrm{Mo}_{2} \mathrm{O}_{7}$, geometrically frustrated magnetism has been shown to provide new ways to engineer such effects with its characteristic noncollinear spin structures leading to unconventional types of the anomalous Hall effect (AHE). The detailed causes of these responses are still the object of debate with mechanisms based on spin structure chirality and the Aharonov-Bohm effect being proposed [3-5]. The paucity of experimental examples with which to test and explore theoretical predictions remains a particular difficulty. The most well-known model systems $\mathrm{Nd}_{2} \mathrm{Mo}_{2} \mathrm{O}_{7}$ and $\mathrm{Pr}_{2} \mathrm{Ir}_{2} \mathrm{O}_{7}$ both have the 3-dimensional pyrochlore structure and display an AHE at low temperature that is related to long- and short-ranged spin-ice-like order of the lanthanide moments, respectively $[3,6]$. The transition metal-based magnet $\mathrm{Fe}_{3} \mathrm{Sn}_{2}$ provides a new direction for study as it is based on a lighter $3 d$ metal and therefore a much weaker spin-orbit interaction (SOI). Its 2-dimensional kagome structure resembles closely the geometry used in many of the theoretical studies. That $\mathrm{Fe}_{3} \mathrm{Sn}_{2}$ displays a giant and unconventional $\mathrm{AHE}$ at room temperature strengthens the possibility that geometrically frustrated magnets could 


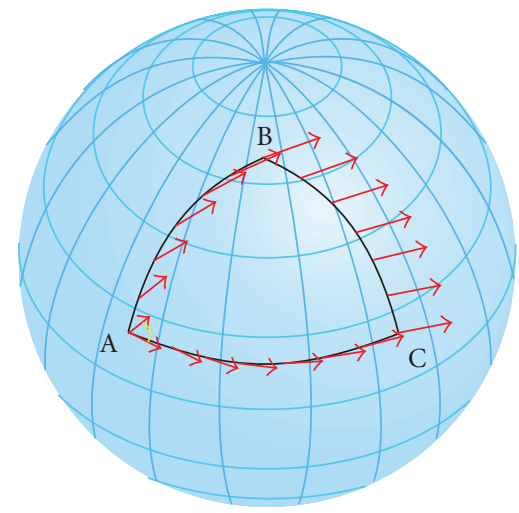

(a)

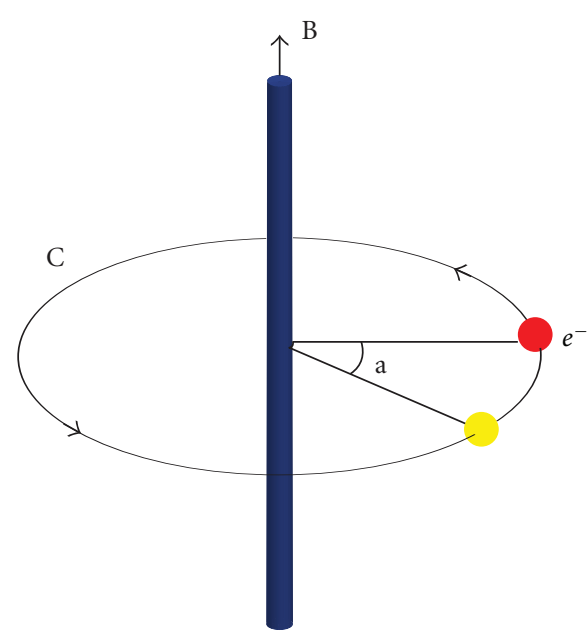

(b)

FIGURE 1: (a) A diagram illustrating parallel transport of a vector constrained to stay tangential to the surface of a sphere: if the red arrow is transported around a closed loop from $\mathrm{A} \rightarrow \mathrm{B} \rightarrow \mathrm{C}$, this constraint causes it to undergo a rotation. The angle of rotation arises from the geometry of the vectors path and is an example of a Berry phase. (b) A schematic of the Aharonov-Bohm effect: an electron processing around an enclosed magnetic field via a loop, C, acquires a Berry phase, a $\left(=\gamma_{n}\right)$, due to coupling of its wavefunction to the magnetic vector potential, despite there being a negligible magnetic field along the path of the electron. The effect is a consequence of the requirement that the electromagnetic potential be invariant with respect to a fixed gauge.

provide access to some of the spintronic properties required for future electronics.

Rather than having only a single origin, the AHE represents a fundamental class of transport properties that are related to an asymmetry in carrier trajectories in solids and the effects of the relativistic spin-orbit interaction. In frustrated lattices it links the physics of RKKY exchange with effects such as Kondo screening and energy scales including the Dzyaloshinskii-Moriya interaction and single-ion anisotropies, to create a rich phase diagram of properties and behaviours. The starting point for understanding the intrinsic AHE came from the theory of Karplus and Luttinger, who showed that spin-orbit coupling in the band structure of ferromagnets can lead to anomalous carrier velocities [7]. More recently, a topological model has been developed where the AHE develops from a Berry curvature and concomitant phase [8].

Empirically, the contributions to the Hall effect are typically written as

$$
\rho_{H}=-\rho_{x y}=R_{0} H+4 \pi R_{S} M,
$$

where $R_{0}$ is the ordinary Hall coefficient, and $R_{\mathrm{S}}$ is the anomalous Hall coefficient that characterises an additional contribution. This equation follows from the observation that the anomalous term is typically proportional to the magnetisation, $M$. However, recent experiments have shown that this monotonic dependence is not necessarily followed and that the AHE can exist in chiral spin systems in the absence of long-range magnetisation and applied magnetic fields, such as in the chiral spin liquid $\operatorname{Pr}_{2} \mathrm{Ir}_{2} \mathrm{O}_{7}$ [9].

This paper focuses on the intrinsic AHE rather than impurity-based mechanisms and summarises the experimental and theoretical models for frustrated ferromagnets based on the pyrochlore and kagome geometries.

\section{The Berry Phase and Its Application to the AHE}

An understanding of the Berry phase and how it arises in frustrated magnets can be obtained from the geometrical concept of parallel transport. This is simply illustrated by considering a vector constrained to lie in a plane tangential to a curved surface (Figure 1(a)). If we transport this vector around a closed loop traced on the surface of a sphere, we find the resulting vector has undergone a rotation by an angle, known as a Berry phase. An analogous result is found in quantum mechanics where the eigenstates of a Hamiltonian are controlled by external parameters, for example, the tracing of a loop in the parameter space of a magnetic field realises the situation of parallel transport and creates a resulting Berry phase [10]. In the majority of cases the Berry phase is zero. However, when a point of degeneracy is enclosed within the loop, a nonzero phase may result.

In simple collinear ferromagnets with a strong spinorbit interaction, singularities in the $k$-space gauge field can appear which are caused by near degeneracy and/or avoided crossing of the conduction bands. A Berry phase results through interaction of a conduction electron with this singularity, which acts as a point of degeneracy. This is a topological understanding of the aforementioned theory of Karplus and Luttinger, where now the anomalous Hall conductivity can be written as the integral of the Berry phase curvature. Recently, however, extraordinary AHE responses have been observed that cannot be explained by this theory.

The Aharonov-Bohm effect is an observable phenomena which results from a Berry phase created in direct (spin) space: when an electron follows a closed path, C, around an enclosed magnetic field, it acquires a phase through interference with the vector potential, despite there being negligible 
field in the path of the electron (Figure 1(b)) [17]. The Berry phase shift

$$
\gamma_{n}(\mathrm{C})=2 \pi N(\mathrm{C}) \frac{q}{h} \Phi
$$

is proportional to the winding number around the magnetic field, $N(\mathrm{C})$, and the flux, $\Phi$, which is given by the amount of magnetic field, $B$, passing through a surface. Similar effects can also occur with itinerant electrons traversing noncollinear, that is, frustrated spin textures. The fictitious magnetic flux resulting from the vector potential couples with the itinerant electrons as if it were real and, as expected, produces a Berry phase and consequent Lorentz force that alters the charge carrier trajectory. There is, however, some debate as to the exact origins of this effect. It has often been suggested in the pyrochlore-based materials that a chiral spin structure gives rise to the fictitious magnetic field, but recently another theory has been proposed based on the arrangement of orbitals of the moment-bearing atoms. The effect that the recent discovery of unconventional AHE in the nonpyrochlore-based frustrated magnets, $\mathrm{Fe}_{3} \mathrm{Sn}_{2}$ and $\mathrm{PdCrO}_{2}$, has on these theories is discussed later.

\section{Singularities in the Band Structure of a Collinear Ferromagnet}

The discovery of exotic physical properties in cuprate-based transition metal oxides has led to a hunt for similar materials containing other transition metal ions. The ruthenates have been a particular focus following the discovery of high- $T_{\mathrm{C}}$ superconductivity in $\mathrm{Sr}_{2} \mathrm{RuO}_{4}$ [18]. Another member of the ruthenates, $\mathrm{SrRuO}_{3}$, was found to be the first $4 d$ ferromagnetic metal and to possess an interplay between itinerant and localised electrons which leads to an unconventional AHE [19]. The origin of this is believed to be a singularity in the Berry curvature, caused by an avoided crossing of the strong spin-orbit split band energies.

$\mathrm{SrRuO}_{3}$ adopts a distorted, pseudo-cubic perovskite structure described in the Pbnm space group $(a=5.5670 \AA$, $b=5.5304 \AA$, and $c=7.8446 \AA$ ) [20], and undergoes a ferromagnetic transition at $T_{\mathrm{C}}=160 \mathrm{~K}$ [21], that is accompanied by a rotation of the $\mathrm{RuO}_{6}$ octahedra [22]. Below $T_{\mathrm{C}}$ a freezing of the rotations causes the lattice parameters to remain effectively constant and for the material to exhibit zero thermal expansion (the Invar effect) in the range $12<T<160 \mathrm{~K}$ [23].

Attempts to characterise the magnetic structure below $T_{\mathrm{C}}$ have been hindered by the sharp reduction in the $4 d$ form factor above $\sim 20^{\circ}(2 \theta)$ and the severe overlap of any observable magnetic Bragg peaks. The ferromagnetic component has been determined from combined high-resolution/highintensity neutron powder diffraction (NPD) as $\mu_{\mathrm{Ru}}=1.63 \pm$ $0.06 \mu_{B}$ at $10 \mathrm{~K}$ [22]. This is lower than expected for the lowspin $\mathrm{Ru}^{4+}(S=1)$ ion where the $4 d^{4}$ electrons are in the $t_{2 \mathrm{~g}}^{4} e_{\mathrm{g}}^{0}$ state, but is in good agreement with electronic structure calculations based on band ferromagnetism $[24,25]$.

Inextricably linked to the complex magnetism in $\mathrm{SrRuO}_{3}$ is the observation of anomalous electrical transport properties. The Hall resistivity, $\rho_{x y}$, shows a strong $T$-dependence
(Figure 2(a)) which cannot be explained by conventional AHE mechanisms. In order to explain this behaviour, the Berry phase mechanism has been adopted. Given that the Hall conductivity, $\sigma_{x y}$, can be written as the integral of the Berry phase curvature of the $t_{2 \mathrm{~g}}$ bands, first principle band structure calculations were performed to map this Berry curvature (Figure 3) [8]. A clear peak is seen at $k_{x y}=(0,0)$, which corresponds to the avoided crossing of the energy bands split by the large spin orbit coupling of $\mathrm{Ru}^{4+}$. This singularity is equivalent to a magnetic monopole accompanied by a Dirac string [8]. When the Fermi energy, $\epsilon_{\mathrm{F}}$, is in the vicinity of this monopole, the electrons are subject to a strong gauge field which creates a large anomalous Hall component. The singularity in the gauge field is clearly represented when plotting first-principle calculations of $\sigma_{x y}$ as a function of the Fermi level position. The constant fluctuation and numerous sign changes as $\epsilon_{\mathrm{F}}$ is shifted are a result of the singular behaviour of the monopoles.

Using this model the anomalous $T$-dependence of $\sigma_{x y}$ can be explained by the exchange splitting of the spinup/spin-down Bloch states, which is proportional to magnetisation, $M$ [8]. Figure 2(b) shows the good agreement of theoretical and experimental values of $\sigma_{x y}$ as a function of $M$ when account is made for the exchange splitting. This model also accounts for studies performed on $\mathrm{Sr}_{1-x} \mathrm{Ca}_{x} \mathrm{RuO}_{3}$, where a decrease in $\mathrm{M}$ with increasing $x$ is found to accompany a quenching of $\sigma_{x y}$ [26].

\section{4. $\mathrm{Nd}_{2} \mathrm{Mo}_{2} \mathrm{O}_{7}-\mathrm{AHE}$ in an Ordered Spin-Ice-Type Structure}

The large anomalous Hall effect discovered in $\mathrm{Nd}_{2} \mathrm{Mo}_{2} \mathrm{O}_{7}$ required revision of existing understanding of intrinsic mechanisms based on the simple band structure and spin-orbit interaction model of the Karplus and Luttinger theory. It was initially thought that the direct space chirality of the frustrated ferromagnetic "spin-ice" structure induced a Berry phase into the wavefunction of a conduction electron, resulting in the observed AHE [3]. However, this theory has now been shown not to agree with experimental calculations of the magnetic interactions $[5,27]$ and a recent alternative explanation has been proposed based on an orbital description of the induced Berry phase $[4,28]$.

$\mathrm{Nd}_{2} \mathrm{Mo}_{2} \mathrm{O}_{7}$ crystallises with cubic symmetry in the $\mathrm{F} d \overline{3} m$ space group $(a=10.4640 \AA)$ [29]. It undergoes a ferromagnetic transition at $T_{\mathrm{C}}=93 \mathrm{~K}$, where the itinerant Mo $4 d$-electron spins order [3]. Upon further cooling below $T=40 \mathrm{~K}$, the localised $\mathrm{Nd} f$-electron spins begin to align antiferromagnetically to the Mo spins. Neutron scattering experiments have accurately determined the magnetic structure and show that the $\mathrm{Nd}$ spins are subject to a strong anisotropy where the easy-axis points to the centre of each tetrahedron, resulting in a sixfold degenerate, chiral "twoin, two-out" spin-ice structure [3]. The coupling between the Mo and Nd tetrahedra, shown in Figure 4, allows the spin chirality of the $\mathrm{Nd}$ tetrahedra to be transferred to the equivalent Mo structure via the $f$ - $d$ exchange interaction $\left(J_{f d} \approx 5 \mathrm{~K}\right)$. Through analysis of a combination of magnetisation and neutron scattering data of the (111) and (200) 


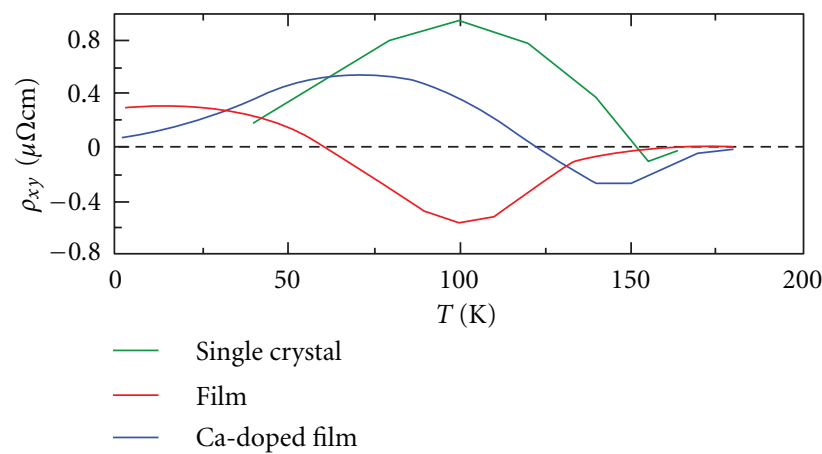

(a)

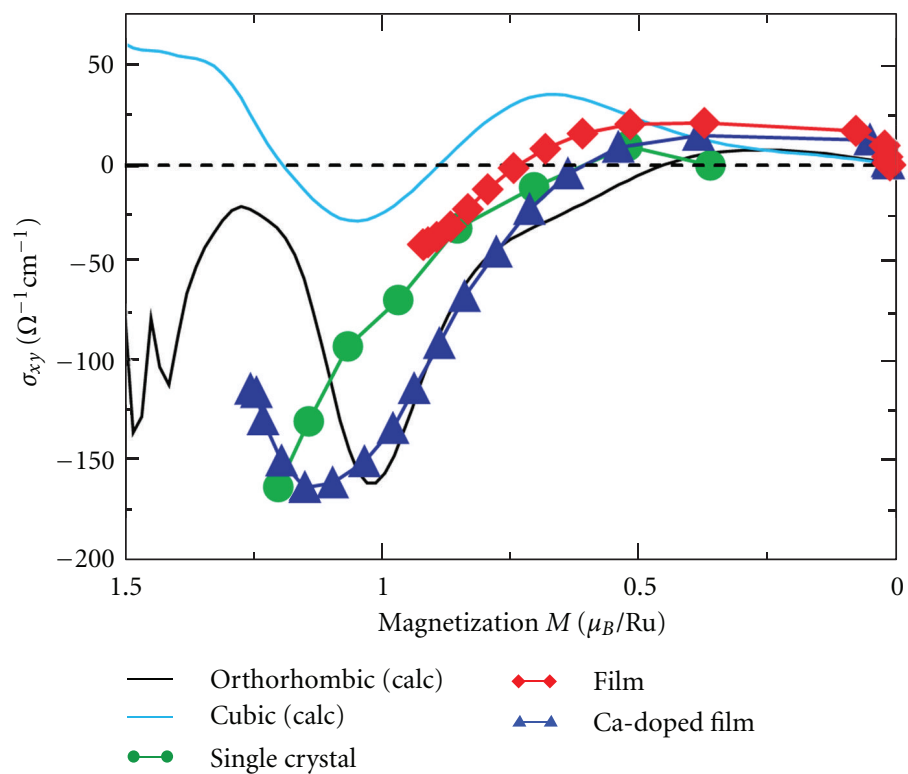

(b)

Figure 2: (a) A plot of transverse resistivity, $\rho_{x y}$, against $T$ for $\mathrm{SrRuO}_{3}$. The strong temperature-dependence cannot be explained by impuritybased extrinsic AHE mechanisms. (b) A comparison with experimental results of first-principle calculations of the Berry curvature and $\sigma_{x y}$ as a function of the magnetization, $M$. The calculations for the orthorhombic phase closely match those of experiment by accounting for the exchange splitting of the bands. All figures are taken from [8].

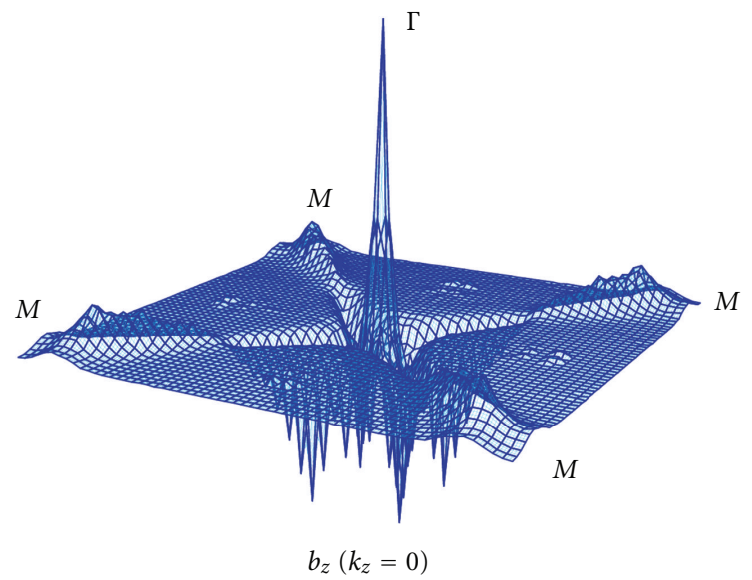

Figure 3: Calculated gauge field strength for $t_{2 \mathrm{~g}}$ bands in $\mathrm{SrRuO}_{3}$ as a function of $\left(k_{x}, k_{y}\right)$ with fixed $k_{z}=0$. The sharp peak at $k_{x}=k_{y}=0$ represents a magnetic monopole at the Fermi surface which strongly affects $\sigma_{x y}$. The figure is taken from [8]. 

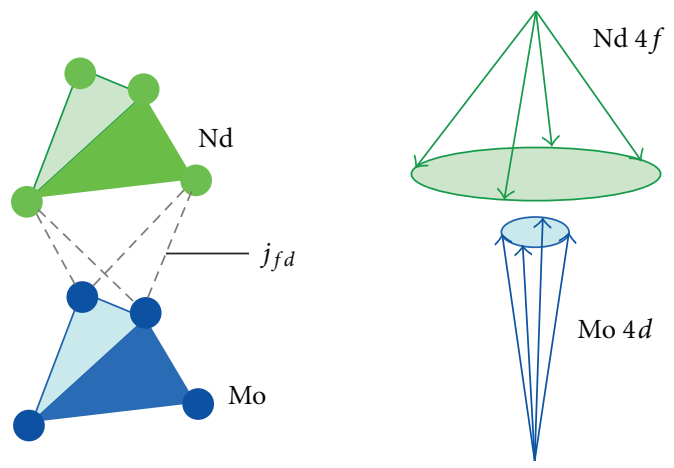

FIgURE 4: Left: the crystal structure of $\mathrm{Nd}_{2} \mathrm{Mo}_{2} \mathrm{O}_{7}$ is made up of Mo and Nd tetrahedra. At $10 \mathrm{~K}$ the spin chirality of the magnetic order on the Nd tetrahedra is transferred to the Mo tetrahedra via the $J_{f d}$ exchange interaction. Right: the magnetic structures of the Nd and Mo tetrahedra form antiparallel "umbrellas". Figure taken from [3].

reflections, the accompanying magnetic structure was shown to be formed of oppositely aligned "umbrellas," Figure 4.

The chirality of this noncoplanar spin structure was originally suggested to be the origin of the observed giant anomalous Hall effect, due to the fictitious field experienced by an itinerant conduction electron. Calculation of $\sigma_{x y}$ from the fictitious magnetic field as a function of the Mo tilting angle, $\theta_{m}$, gives a relation $\sigma_{x y} \propto \theta_{m}^{2}$ and the observed value of $\left|\sigma_{x y}\right|$ can be reproduced by an angle of $\theta_{m}=4^{\circ}$ [3]. Scaling of $\rho_{x x}$ with the anomalous Hall resistivity arising from the Mo spin chirality $\left(\rho_{\mathrm{H}}^{\chi}\right)$ in Nb-doped $\mathrm{Nd}_{2} \mathrm{Mo}_{2} \mathrm{O}_{7}$, $\mathrm{Nd}_{2}\left(\mathrm{Mo}_{1-x} \mathrm{Nb}_{x}\right)_{2} \mathrm{O}_{7}$, further distinguishes the chirality mechanism from an increase in the SOI of the Mo site upon doping (Figure 5) [11]. However, this analysis is based on a simple decomposition of the anomalous Hall effect into spin chirality $\left(\rho_{\mathrm{H}}^{\chi}\right)$ and spin-orbit $\left(\rho_{\mathrm{H}}^{\mathrm{SO}}\right)$ induced components

$$
\rho_{\mathrm{H}}(H)=R_{0} H+\rho_{\mathrm{H}}^{\chi}(H)+\rho_{\mathrm{H}}^{\mathrm{SO}}
$$

with no indication given of the form of this SOI contribution.

There do, however, appear to be problems with the chirality mechanism [5]. Single crystal neutron scattering experiments performed in magnetic fields allowed determination of several magnetic parameters, such as exchange constants for Mo-Mo, Nd-Mo, and Nd-Nd couplings. These parameters were used to calculate the fictitious magnetic flux as a function of $H$ and $T$ and also to deduce the Hall resistivity, $\rho_{\mathrm{H}}$ [5]. These results cannot explain the experimental trends and, as such, the chirality mechanism cannot solely explain the observed AHE (Figure 6). This discrepancy with previous theories could be due to different Mo tilting angles used $\left(\theta_{m}=1^{\circ}\right.$ in [5] $)$ and therefore Mo-Nd interaction energies. Accurate determination of these demands single crystal samples $[5,27,30]$.

Another mechanism proposed to account for the observed AHE in $\mathrm{Nd}_{2} \mathrm{Mo}_{2} \mathrm{O}_{7}$ and to overcome the deficiencies of the chirality mechanism is that based on a Berry phase induced by a complex $t_{2 g}$ orbital arrangement $[4,28]$. In this mechanism the Berry phase arises when an electron moves around a circuit made up of the orbitals of the tetrahedral motif. The resulting fictitious magnetic flux, $\Phi_{\text {orb }}$, is linear with respect to $\theta$, unlike the chirality-driven mechanism where the fictitious magnetic flux is quadratic in the spin-canting angle, $\Phi_{\text {spin }} \propto \theta_{m}^{2}$. This $\theta_{m}$-dependence reproduces the experimental results well, with the anomalous Hall resistivity relation given by $\rho_{\mathrm{H}} \sim 4 \pi R_{\mathrm{S}} M_{\mathrm{Z}}^{\mathrm{Mo}}+4 \pi R_{\mathrm{S}}^{\prime} M_{\mathrm{Z}}^{\mathrm{Nd}}$, where the second term represents the unconventional AHE due to the noncollinear spin configuration of the localised $\mathrm{Nd}$ moments by the orbital Aharonov-Bohm effect $[4,28]$. Furthermore, the nonzero values of the anomalous Hall coefficient obtained at $\theta_{m}=0$ and $90^{\circ}$, where the spin chirality term is zero, indicate that the spin chirality mechanism is not the dominant mechanism. This methodology has also been used to explain the AHE in another pyrochlore, $\operatorname{Pr}_{2} \mathrm{Ir}_{2} \mathrm{O}_{7}$, and will be discussed in the next section.

\section{5. $\operatorname{Pr}_{2} \mathrm{Ir}_{2} \mathrm{O}_{7}-\mathrm{AHE}$ in a Chiral Spin Liquid}

Following the initial studies of $\mathrm{Nd}_{2} \mathrm{Mo}_{2} \mathrm{O}_{7}$, there was a demand for further examples of metallic pyrochlore materials. The pyrochlore iridates, $R_{2} \operatorname{Ir}_{2} \mathrm{O}_{7}$, were likely candidates with large rare-earth ions $(R=\mathrm{Eu}, \mathrm{Sm}, \mathrm{Nd}$ or $\mathrm{Pr})$ being shown to suppress the metal-insulator transitions seen with lighter rare-earths [31]. Particular interest was given to $\mathrm{Pr}_{2} \mathrm{Ir}_{2} \mathrm{O}_{7}$ as no magnetic ordering is seen to the lowest measured temperatures. Despite this $\operatorname{Pr}_{2} \mathrm{Ir}_{2} \mathrm{O}_{7}$ still displays a large anomalous Hall effect even in zero field [9]. As with $\mathrm{Nd}_{2} \mathrm{Mo}_{2} \mathrm{O}_{7}$ initial explanations revolved around the chirality mechanism originating from a chiral spin-liquid state. However, very recent explanations using the orbital description appear to give a better explanation of the results [4].

Whilst adopting the same pyrochlore structure as $\mathrm{Nd}_{2} \mathrm{Mo}_{2} \mathrm{O}_{7}$, the main crystallographic difference with $\mathrm{Pr}_{2} \mathrm{Ir}_{2} \mathrm{O}_{7}$ is that the $\mathrm{B}$-site is occupied by a $5 d$, rather than $4 d$, transition metal. $\left(\operatorname{Pr}_{2} \mathrm{Ir}_{2} \mathrm{O}_{7}\right.$ is also described in the $\mathrm{F} d \overline{3} m$ space group and has the lattice parameter $a=10.396 \AA$. No structural transition is observed at any temperature.)

Magnetic susceptibility measurements show an antiferromagnetic Weiss temperature, $\theta_{\mathrm{W}}=-20 \mathrm{~K}$, due to the RKKY interaction, but no long-range ordering transition is seen down to $70 \mathrm{mK}$; there is only a partial freezing at $120 \mathrm{mK}$. The [111] Ising-like $\operatorname{Pr}^{3+} 4 f$ spins remain paramagnetic due to the partial screening from the Kondo effect of the Ir $5 d$-conduction bands [32]. The screening renormalises the 


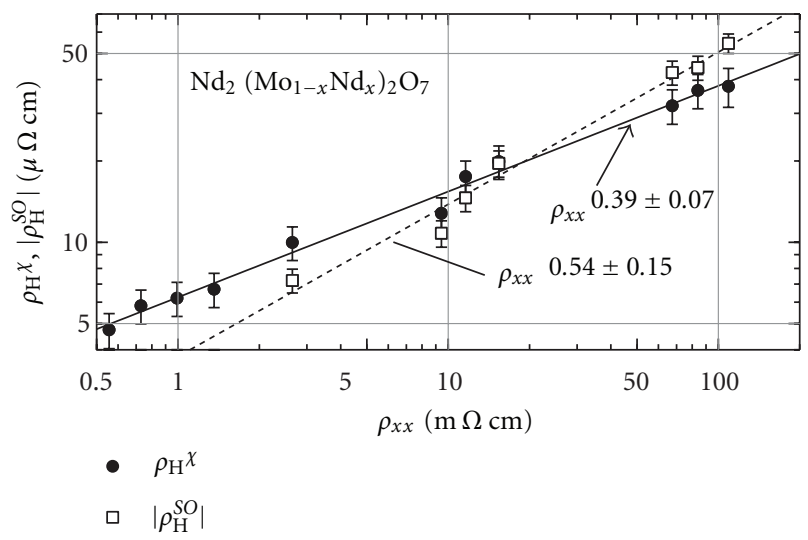

FIgURE 5: A plot of the relationship in $\mathrm{Nd}_{2}\left(\mathrm{Mo}_{1-x} \mathrm{Nb}_{x}\right)_{2} \mathrm{O}_{7}$ between the longitudinal resistivity, $\rho_{x x}$, and the anomalous Hall components arising from the spin chirality, $\rho_{H}^{\chi}$, and from the SOI, $\rho_{H}^{\mathrm{SO}}$, taken from [11].

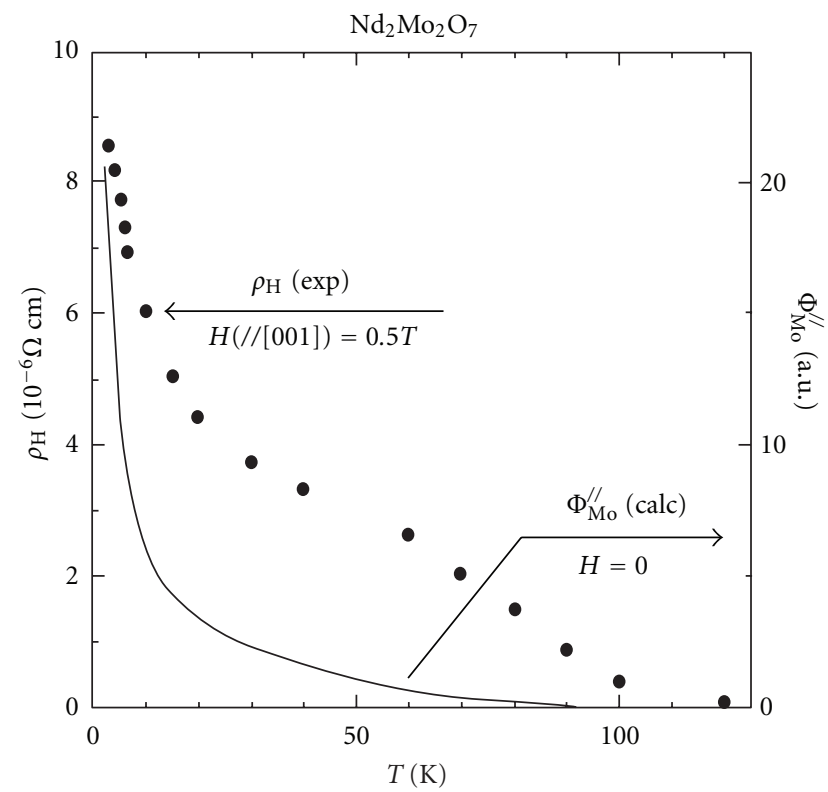

FIgure 6: A plot of experimental Hall resistivity, $\rho_{H}$, in $\mathrm{Nd}_{2} \mathrm{Mo}_{2} \mathrm{O}_{7}$ as a function of temperature. The mismatch between the experimental values and the fictitious magnetic flux, $\Phi_{\mathrm{Mo}}$, calculated from the chirality of the magnetic structure indicates that the chirality mechanism is not the majority component of the AHE. The figure is taken from [5].

antiferromagnetic interaction to a value of $\theta_{\mathrm{W}}=-1.7 \mathrm{~K}$. Below this temperature, spin-liquid behaviour is observed with a small quasistatic moment $\left(0.1 \mu_{B}\right)$ present, most likely due to hyperfine enhanced ${ }^{141} \mathrm{Pr}$ nuclear moments, as observed in $\mu_{\mathrm{SR}}$ [33]. Furthermore, a metamagnetic transition occurs when a field is applied along the [111] direction due to a coupling of the field to a ferromagnetic exchange term, $J \sim 1.4 \mathrm{~K}[6]$. The lack of long-range magnetic order causes the Ir moments to experience a local exchange field, $\mathbf{h}_{i}$, that is composed only of contributions from the local short-range ordered Pr moments. These lead to a tilting angle of the Ir moments, $\theta_{\text {Ir }}$, that is much larger than that of the Mo in $\mathrm{Nd}_{2} \mathrm{Mo}_{2} \mathrm{O}_{7}$.

The anomalous Hall resistivity, $\rho_{x y}$, begins a gradual increase below $T^{*}=20 \mathrm{~K}$ until $\left|\theta_{\mathrm{W}}\right|=1.7 \mathrm{~K}$, when it increases sharply, reaching a value of $\sim 3 \mu \Omega \mathrm{cm}$ as $T \rightarrow 0$ (Figure 8) [9]. Below $\left|\theta_{\mathrm{W}}\right|, R_{\mathrm{S}}$ increases logarithmically, while $\rho_{x x}$ remains constant; neither of these follow the commonly observed relationships. Furthermore, the field-dependence of $\sigma_{x y}$ shows a large isotropic increase until a critical value of the field is reached, $B_{C} \approx 0.7 \mathrm{~T}$, after which there is strong anisotropic and nonmonotonic $B$-dependence, with the [100] direction reaching a very large value of $\sim 30 \Omega^{-1} \mathrm{~cm}^{-1}$, but the [111] direction decreasing at higher fields.

Explanations of this AHE behaviour have revolved around the idea of a "chiral spin liquid" consisting of a spinice "2-in, 2-out" spin structure. This model was developed following the observation of the onset of a zero-field AHE below $T_{\mathrm{H}} \approx 1.5 \mathrm{~K}$ in the absence of any long-range order 


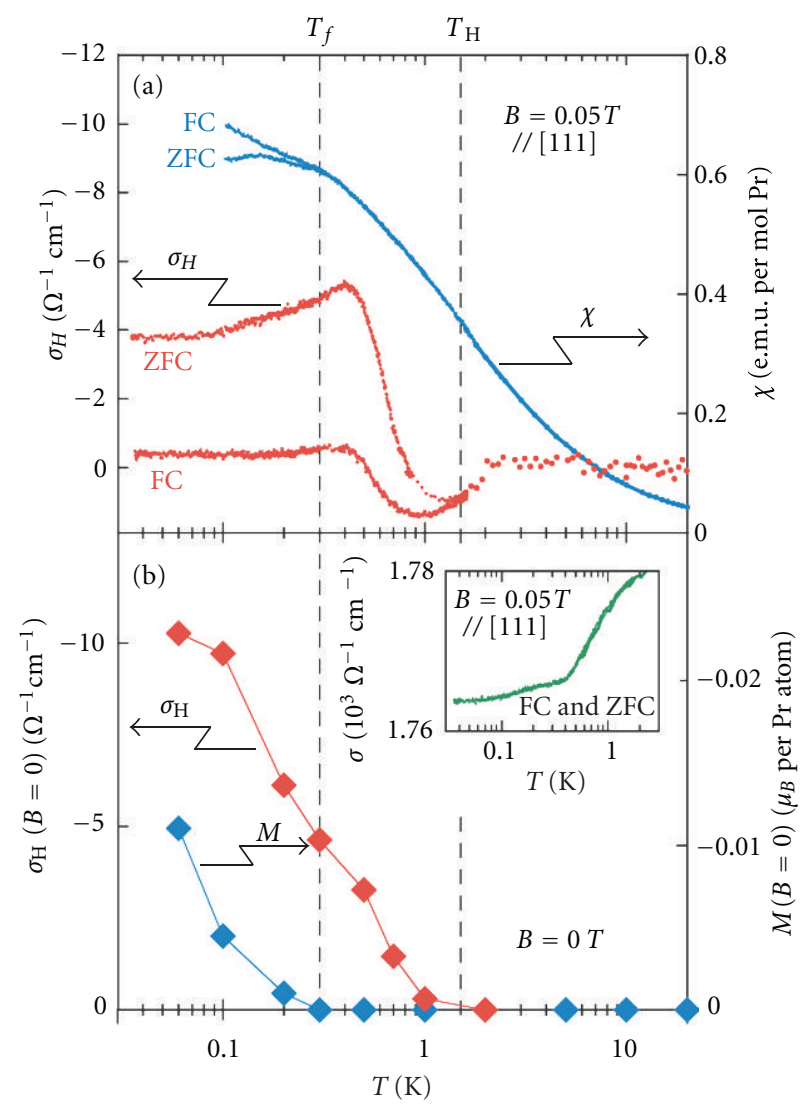

(c)

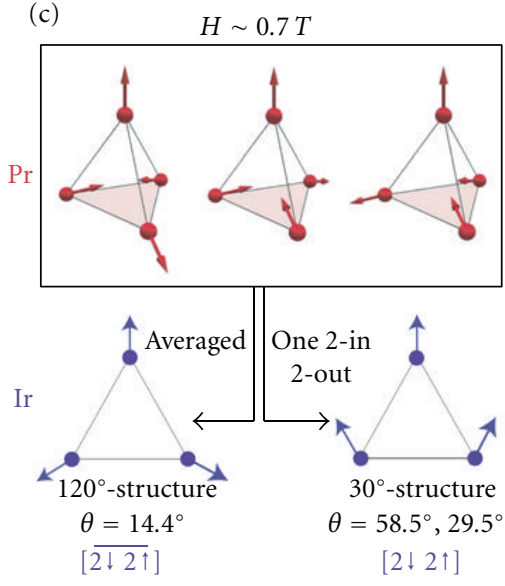

$H>0.7 T$
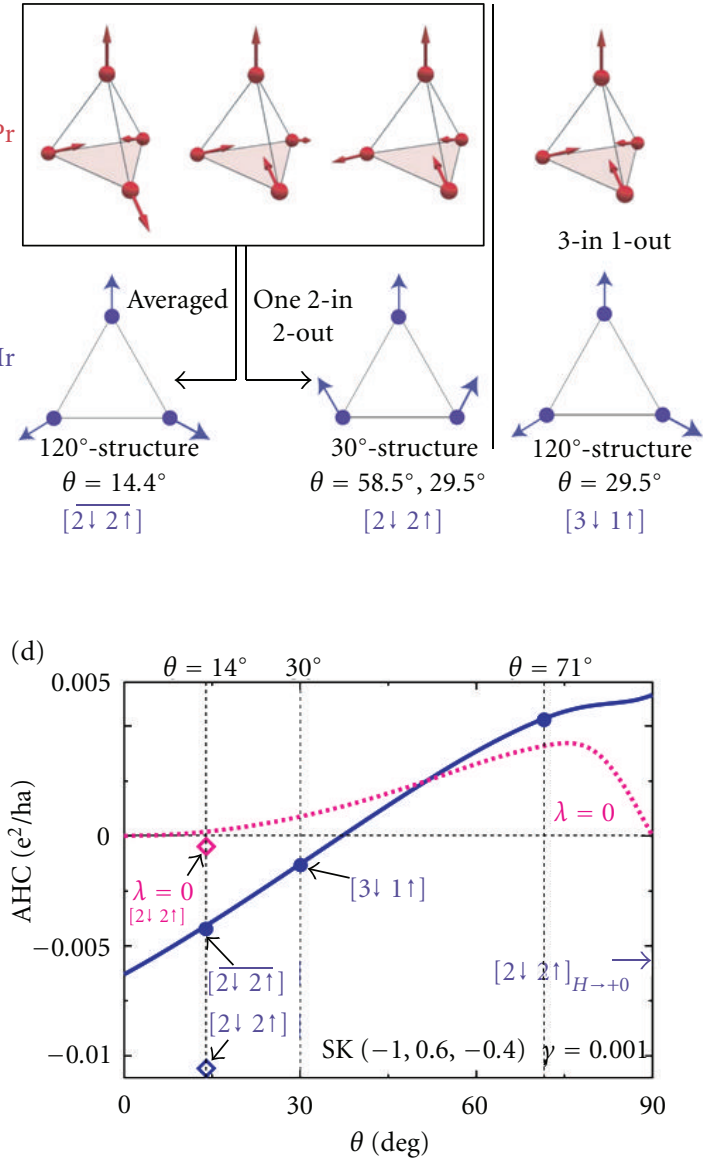

Figure 7: (a) Zero-field-cooled/field-cooled temperature dependence of the Hall conductivity (left axis) and magnetic susceptibility (right axis), measured in a field of $B=0.05 \mathrm{~T}$ along the [111] direction of $\operatorname{Pr}_{2} \operatorname{Ir}_{2} \mathrm{O}_{7}$ single crystals. Bifurcation of $\sigma_{\mathrm{H}}$ at $T_{\mathrm{H}}$ occurs above any magnetic ordering temperature observed in $\chi$. (b) Zero-field temperature dependence of $\sigma_{\mathrm{H}}$ and magnetisation, $M$. A spontaneous Hall conductivity occurs at $T_{\mathrm{H}}$ in the absence of any magnetic moment, which does not appear until below $T_{f}$. Figures are from [6]. (c) The induced Ir spin structures from the "2-in, 2-out" and "3-in, 1-out" Pr tetrahedra in $\operatorname{Pr}_{2} \operatorname{Ir}_{2} \mathrm{O}_{7}$, taken from [4]. (d) Calculated $\theta$-dependence of the anomalous Hall coefficient for various spin structures in $\operatorname{Pr}_{2} \operatorname{Ir}_{2} \mathrm{O}_{7}$, using the SK $d$ - $d$ hopping integrals shown. The nonzero values obtained at $\theta=0$ and $90^{\circ}$, where the spin chirality term is zero, indicate that the spin chirality mechanism is not the dominant mechanism, taken from [4].

or magnetic component (i.e., $T_{\mathrm{H}}>T_{f}$ ) [6]. A bifurcation of field-cooled (FC) and zero-field-cooled (ZFC) data of $\sigma_{\mathrm{H}}$ is also seen at $T_{\mathrm{H}}$ in a field of $0.05 \mathrm{~T}$ applied along [111] (Figures 7(a) and 7(b)). The disappearance of this bifurcation at $B_{\mathrm{C}}$ is argued to indicate a transition from a "2in, 2-out" to a "3-in, 1-out" structure [6]. Field-dependent hysteresis around zero field along [111], which is severely reduced along the [100] and [110] directions, could also be explained by stabilisation of the " 3 -in, 1-out" structure [12]. The coincidental onset temperature, $T_{\mathrm{H}}$, with the calculated ferromagnetic component, $J_{f f} \sim 1.4 \mathrm{~K}$, provides further evidence of a non-zero chirality state which is able to induce the AHE. However, accurate determination of the ferromagnetic component is required, as this effect could be a result of further-neighbour interactions.

An alternative explanation, as mentioned in the previous section, lies in the orbital Aharonov-Bohm theory. Calculations of the $\theta_{\mathrm{Ir}}$-dependence of the AHE for various Ir spin structures were done using direct $\mathrm{d}-\mathrm{d}$ hopping integrals from
Slater-Koster (SK) parameters and values of the atomic SOI of $\lambda=3000 \mathrm{~K}$ to represent the orbital $\mathrm{AB}$ effect; $\lambda=0 \mathrm{~K}$ is correspondingly used to model the spin chirality term (Figures 7(c) and 7(d)) [4]. The orbital Aharonov-Bohm effect is shown to dominate over the spin chirality term and the calculated $\theta$-dependence of the AHE is found to agree with experiment as the structure changes from the " 2 -in, 2 -out" to the " 3 -in, 1-out" structure. The low- $T$ hysteresis is explained by adopting an achiral spin structure based on $J \gg\left|\mathbf{h}_{i}\right|$, which gives a finite anomalous Hall coefficient. Reversing the spin structure provides a simple explanation of the observed hysteresis.

\section{Room Temperature Giant AHE in a 2-Dimensional Noncollinear Ferromagnet}

The intermetallic compound $\mathrm{Fe}_{3} \mathrm{Sn}_{2}$ provides a new type of frustrated metal that complements the previously discussed pyrochlores. The structure is formed of 2-dimensional 


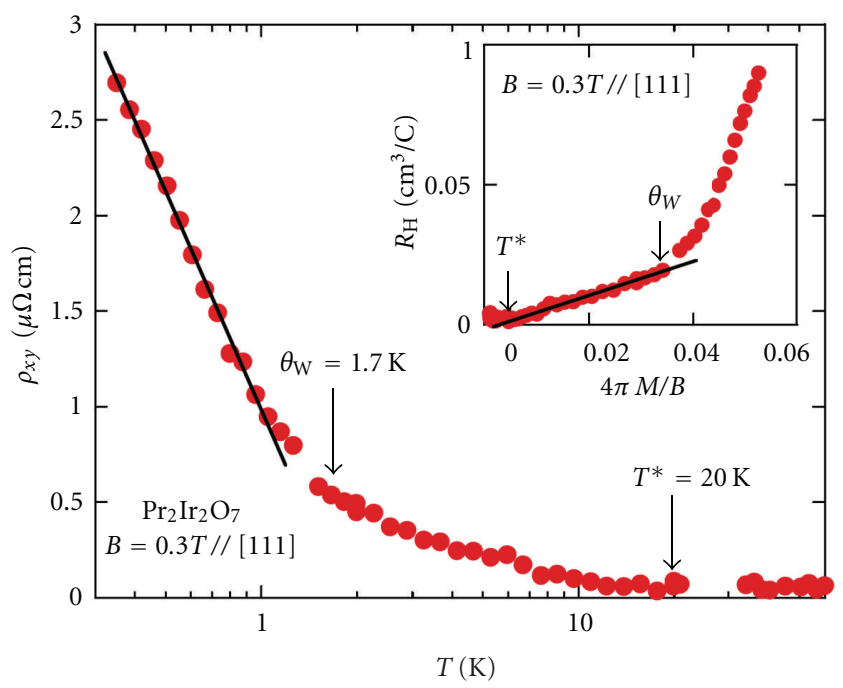

(a)

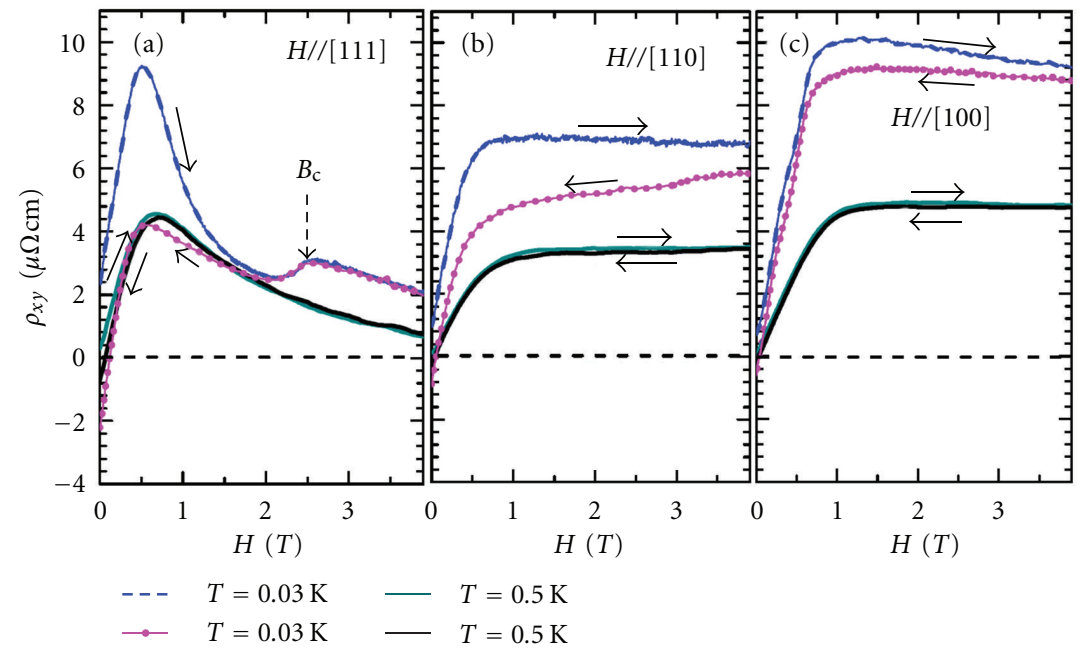

(b)

Figure 8: (a) A plot of anomalous Hall resistivity, $\rho_{x y}$, against $T$, measured in a field of $0.3 \mathrm{~T}$ along the [111] direction of $\mathrm{Pr}_{2} \mathrm{Ir}_{2} \mathrm{O}_{7}$ single crystals. A clear increase is seen below $T^{*}=20 \mathrm{~K}$ before exponential growth below the Weiss temperature, $\left|\theta_{\mathrm{W}}\right|=1.7 \mathrm{~K}$. The inset shows the reverse behaviour for the ordinary Hall coefficient, $R_{\mathrm{H}}$, under the same conditions. The figure is taken from [9]. (b) Field-dependence of the anomalous Hall resistivity, $\rho_{x y}$, with the field applied along the (a) [111], (b) [110], and (c) [100] directions of $\operatorname{Pr}_{2} \operatorname{Ir}_{2} \mathrm{O}_{7}$ single crystals. The pronounced hysteresis along the [111] direction, suggested to host the "3-in, 1-out" spin structure, is severely reduced along [110] and [100] due to the stabilisation of the "2-in, 2-out" structure. Figure from [12].

kagome planes and the itinerant electrons are from the $3 d$ orbitals of $\mathrm{Fe}$, in contrast to heavier $\mathrm{Nd}$, Ir, and $\mathrm{Ru}$ of the aforementioned pyrochlores. The 2-dimensionality of the lattice and weaker spin orbit interaction of the $3 d$ electrons should therefore result in a band structure that is significantly different from those of the previous materials. It therefore provides a very different system with which to test the proposed intrinsic mechanisms of the AHE. Furthermore, giant values of the AHE are found at room temperature, allowing the possibility of application into spintronics devices [14].
The crystal structure of $\mathrm{Fe}_{3} \mathrm{Sn}_{2}$ (Figure 9) is described in the space group $\mathrm{R} \overline{3} \mathrm{~m}$. Bilayer planes of Fe and $\mathrm{Sn}$ atoms are stacked along the hexagonal [001] direction, separated by planes of crystallographically inequivalent $\mathrm{Sn}$ atoms [34]. The bilayer planes consist of two separate Fe kagome lattices offset along [001], with an Sn residing within each kagome hexagon. While the magnetic Fe atoms order ferromagnetically at $T_{\mathrm{C}}=640 \mathrm{~K}\left(\mu_{\mathrm{Fe}} \approx 2.2 \mu_{\mathrm{B}}\right)$ along the $c$-axis, competing interactions at lower temperatures cause a nontrivial magnetic structure [13]. Magnetic refinement of neutron powder diffraction data reveals a symmetry relaxation 


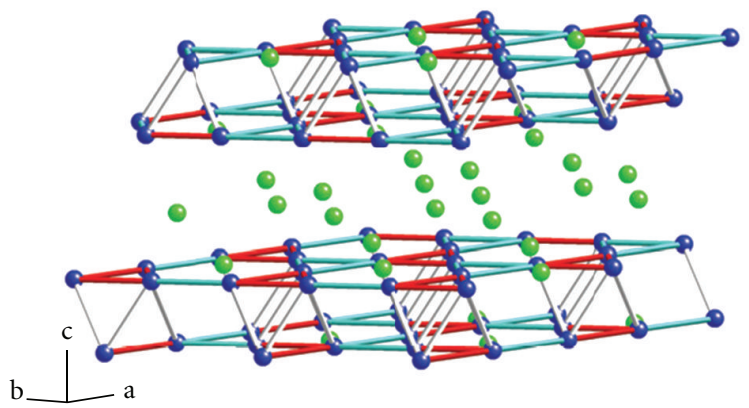

(a)

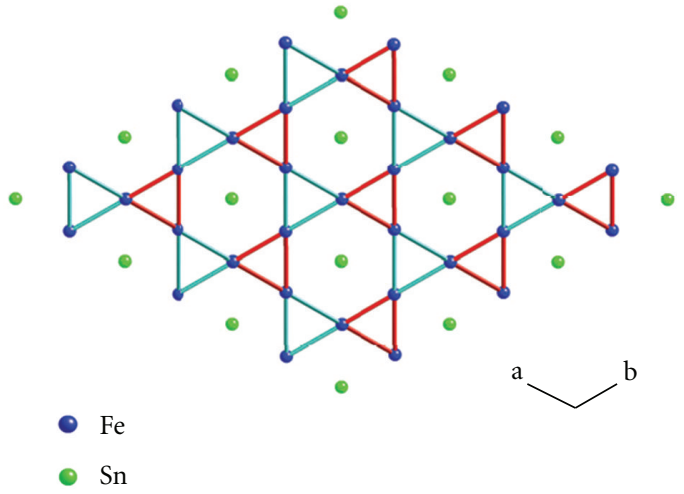

(b)

Figure 9: (a) Crystal structure of $\mathrm{Fe}_{3} \mathrm{Sn}_{2}$ showing the kagome bilayers separated by layers of $\mathrm{Sn}$ atoms. (b) View perpendicular to the abplane showing an Fe kagome layer: the red and blue Fe-Fe bonds denote two different sizes of equilateral triangles. The Sn atoms within the bilayers occupy positions at the centre of the kagome hexagons to form a triangular network. The figure is taken from [13].

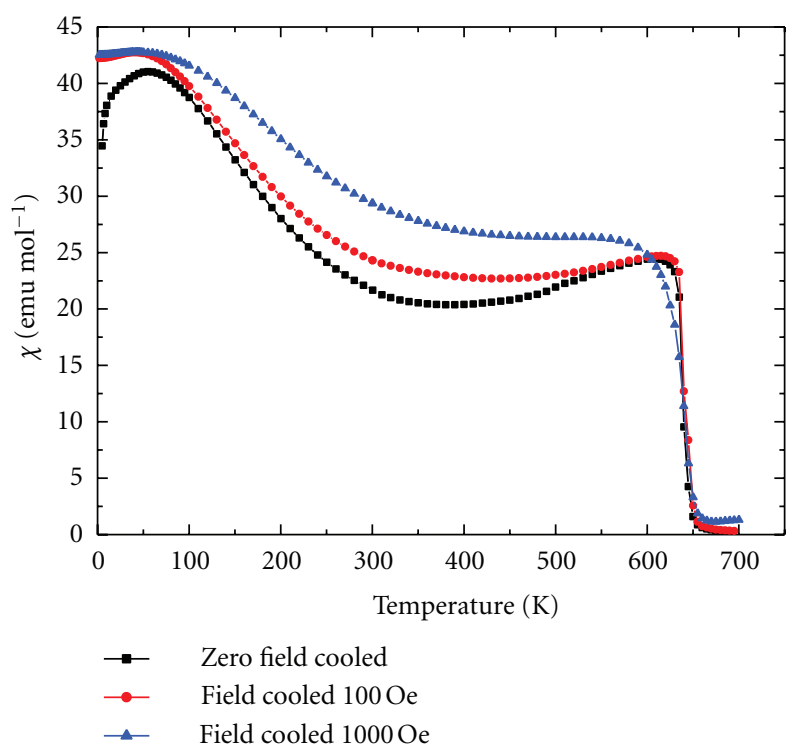

(a)

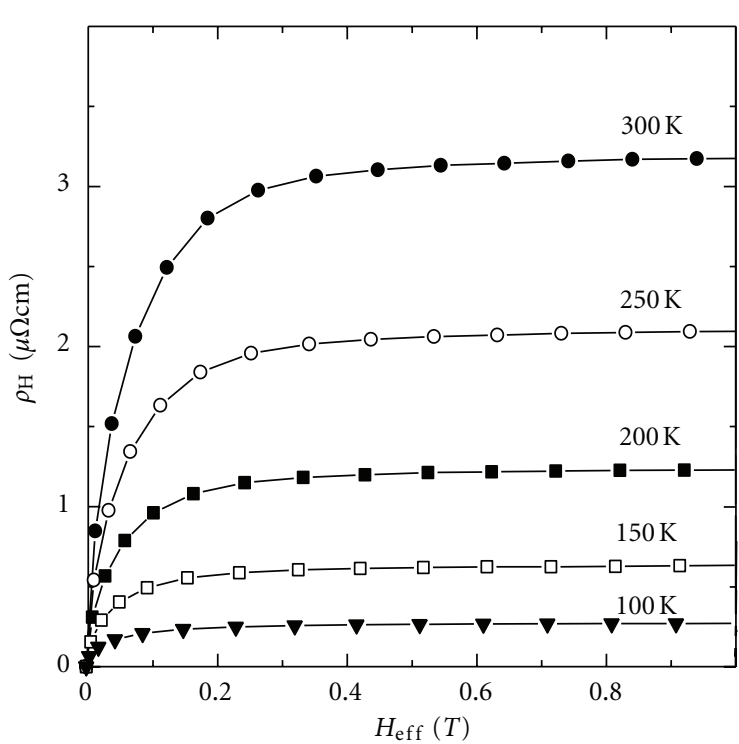

(b)

FIGURE 10: (a) Zero-field-cooled and field-cooled magnetic susceptibility, $\chi$, of powdered $\mathrm{Fe}_{3} \mathrm{Sn}_{2}$. Upon cooling below the Curie temperature, $T_{\mathrm{C}} \approx 650 \mathrm{~K}$, the moments order ferromagnetically along the hexagonal $c$-axis. Below $T \approx 350 \mathrm{~K}$, the moments then rotate into the $a b$-plane. During this transition, the moments become noncollinear, shown by the gradual increase in $\chi$. The broad maximum at $T \approx 80 \mathrm{~K}$ indicates a transition to a spin glass state which coexists with an ordered ferromagnetic moment now lying largely in the $a b$-plane, taken from [13]. (b) Field-dependence of $\rho_{x y}$ at various temperatures. The dependency is similar to that observed for $M$. The large room temperature saturated value of $3.2 \mu \Omega \mathrm{cm}$ demonstrates the potential for geometrically frustrated ferromagnets as materials for spintronics devices, taken from [14].

below $T \approx 350 \mathrm{~K}$ that allows a noncollinear magnetic component to develop as the moments rotate out of the [001] direction and towards the kagome plane. This noncollinearity and competing transitions are clear indications of competing energy scales and magnetic frustration [13].

Magnetic susceptibility data highlights a second transition $(T \approx 80 \mathrm{~K})$ into a reentrant spin glass phase, in which the moments lie largely within the kagome, (001), plane [13] (Figure 10(a)). To add further complexity to the picture, very recent single crystal susceptibility data has revealed a fielddependent discontinuous transition during the moment rotation regime at $T \approx 150 \mathrm{~K}$ [35]. Accompanying this transition is a localised "melting" of the dendritic domain structure, evidenced through magnetic force microscopy (MFM) imaging [35].

The Hall resistivity of $\mathrm{Fe}_{3} \mathrm{Sn}_{2}$ shows different behaviour from the previously discussed materials (Figure 10(b)). The field-dependence of $\rho_{x y}$ follows that of the magnetisation, $M$ : a rapid increase is seen from $H_{\text {eff }}=0$ to $\sim 0.7 \mathrm{~T}$, followed by saturation at higher fields [14]. The saturation value of $\rho_{x y}$ increases with temperature, in contrast to $\mathrm{Nd}_{2} \mathrm{Mo}_{2} \mathrm{O}_{7}$ and $\mathrm{Pr}_{2} \mathrm{Ir}_{2} \mathrm{O}_{7}$ reaching a value of $3.2 \mu \Omega \mathrm{cm}$ at $300 \mathrm{~K}$, the highest 


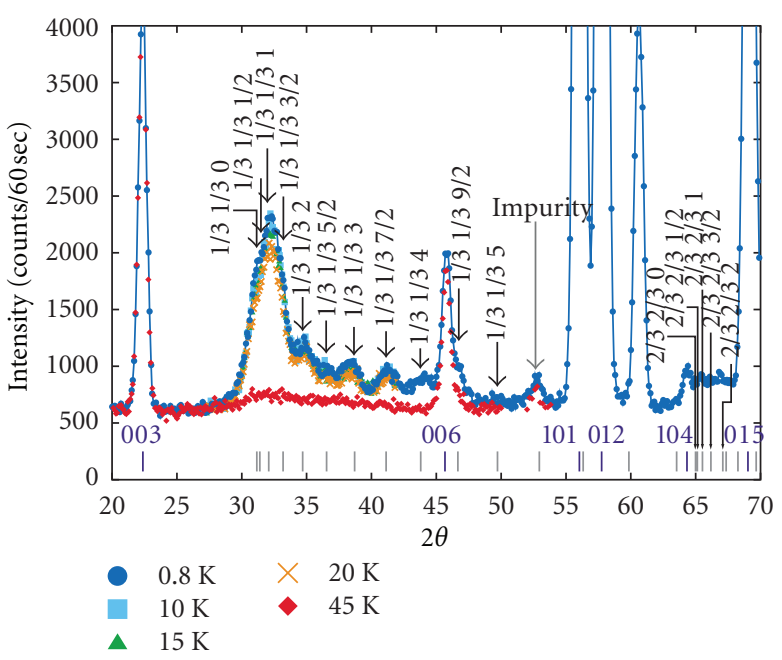

(a)

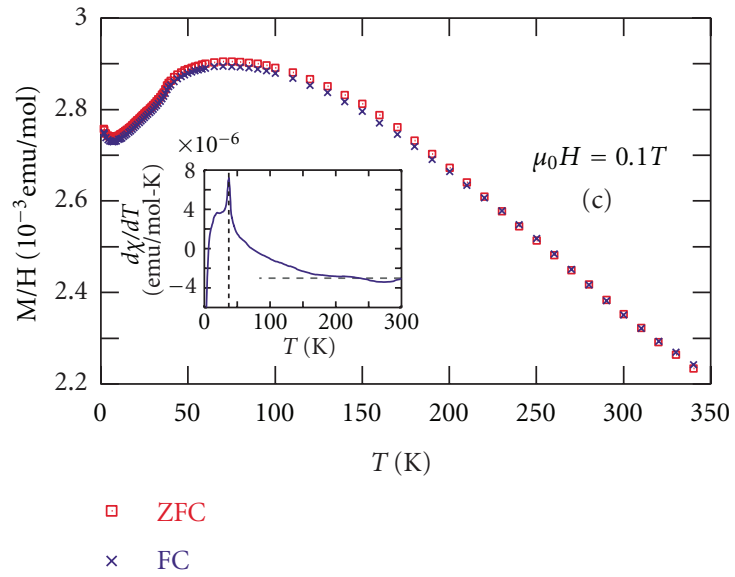

(b)

Figure 11: (a) Powder neutron diffraction patterns of $\mathrm{PdCrO}_{2}$ collected at various temperatures with neutrons of wavelength $\lambda=2.3475 \AA$. The broadness of the magnetic peaks indicates that the correlations are short ranged. Note the observation of $\left(\begin{array}{lll}1 / 3 & 1 / 2 & 1 / 2\end{array}\right)$ Bragg peaks. (b) Temperature dependence of the field-cooled and zero-field-cooled magnetic susceptibility, $\chi$, measured in a field of $0.1 \mathrm{~T}$. The inset shows a clear anomaly in the derivative $d \chi / d T$ at $T_{\mathrm{N}}=40 \mathrm{~K}$. Both figures are taken from [15].

temperature measured. The coincidence of the $M$ and $\rho_{x y}$ saturation fields indicates that the dominant Hall contribution is from the anomalous component. Extracted values of the anomalous Hall coefficient, $R_{\mathrm{S}} \approx 10^{-11}-10^{-10} \Omega \mathrm{cmG}^{-1}$, are one to two orders of magnitude larger than those of $\mathrm{Fe}$ and $\mathrm{Ni}$, and the residual component as $\mathrm{T} / \mathrm{T}_{\mathrm{C}} \rightarrow 0 \mathrm{~K}$ suggests a negligible extrinsic mechanism [14]. The unconventional nature of the AHE is confirmed by the scaling of $R_{\mathrm{S}}$ to $\rho_{x x}$, which gives an exponent $R_{\mathrm{S}} \propto \rho_{x x}^{3.15}$, different to any known AHE mechanism.

The unconventional Hall behaviour is assumed to originate from the nontrivial, frustrated magnetic structure, though whether this is a result of an induced Berry phase remains to be determined. Band structure calculations are required to determine whether an Aharonov-Bohm effect based on the $3 d \mathrm{Fe}$ orbitals occurs, a situation that would be quite different from the heavy metal containing pyrochlores.

\section{7. $\mathrm{PdCrO}_{2}-\mathrm{AHE}$ in an 120 Degree Antiferromagnetic Spin Structure}

Materials with antiferromagnetically interacting spins on a 2dimensional triangular-lattice have attracted much attention due to their magnetic frustration and initial proposals of possible spin liquid ground states. $\mathrm{PdCrO} 2$ provides a rare example of a magnetic triangular-lattice metal to probe transport properties in the presence of itinerant and localised frustrated spins.

$\mathrm{PdCrO}_{2}$ crystallises in the $\mathrm{R} \overline{3} m$ space group with lattice parameters $a=2.9320 \AA$ and $c=18.087 \AA$ [36]. Triangular layers of $\operatorname{Pd}\left(\mathrm{O}_{2}\right)$ and $\operatorname{Cr}\left(\mathrm{O}_{6}\right)$ are alternately stacked along the $c$-axis. The magnetic susceptibility (Figure 11(b)) shows Curie-Weiss behaviour until $T \sim 300 \mathrm{~K}$, below which a broad hump is seen at $T \sim 60 \mathrm{~K}$ indicating short-range order, followed by a gradual decrease [37]. A weak anomaly is then seen at $T_{\mathrm{N}}=40 \mathrm{~K}$, which also appears more prominently in specific heat data, before a final upturn below $T \approx 10 \mathrm{~K}$ [15]. The Weiss temperature, $\theta_{\mathrm{W}}=-500 \mathrm{~K}$, indicates the presence of strong antiferromagnetic interactions, while the effective $\mathrm{S}=(3 / 2) \mathrm{Cr}^{3+}$ moment is $4.1 \mu_{B}$.

Powder neutron diffraction, measured at 8,75 and $150 \mathrm{~K}$, shows only diffuse magnetic scattering at $2 \theta \approx 34^{\circ}$, which has been used to suggest the presence of local $120^{\circ}$ spin ordering (Figure 11(a)). Unconventional behaviour of the Pd $4 d^{9}$ electrons is seen in the Hall resistivity, $\rho_{x y}$, which shows nonlinear field-dependence below $T=40 \mathrm{~K}$, developing into a broad hump at $T^{*}=20 \mathrm{~K}$ (Figure 12(a)) [16]. Given this result, calculating $R_{\mathrm{S}}$ using the equation

$$
R_{\mathrm{S}}(H, T)=\frac{\left[\rho_{x y}(H, T)-R_{0} B\right]}{4 \pi M(H, T)}
$$

shows that $R_{\mathrm{S}}$ only becomes field-dependent below $T^{*}=$ $20 \mathrm{~K}$ and also retains a large, finite value at low temperatures (Figure 12(b)). Furthermore, $\theta_{x y}$ is shown to be dominated by an unconventional mechanism at low temperatures. With such similarly anomalous results to the pyrochlore materials, it is logical to probe the suitability of the spin chirality and orbital Berry phase mechanisms. However, further experiments are needed to determine any subtle changes to the low temperature magnetic structure and calculate the electronic band structure.

\section{Conclusion}

A theoretical consensus on the origins of extraordinary types of Anomalous Hall Effect (AHE) in conducting magnets is far from being reached. To help achieve this we have summarised 


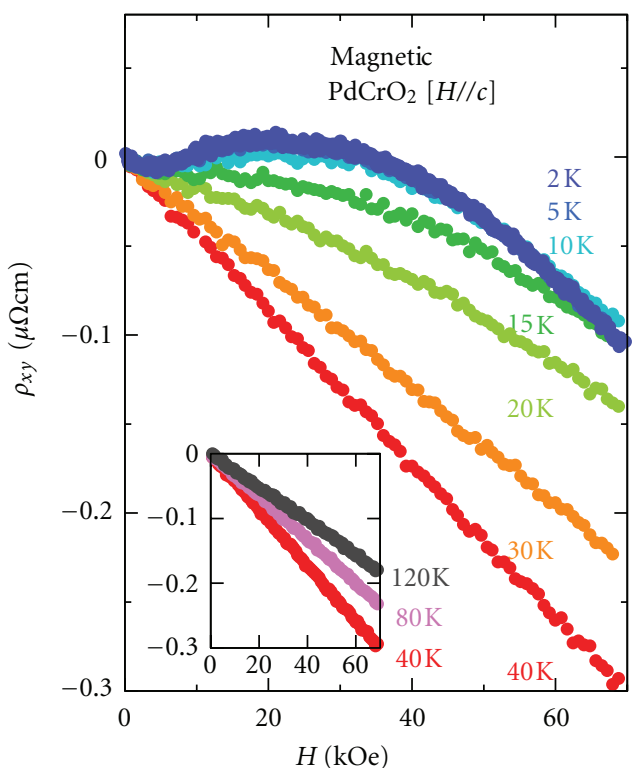

(a)

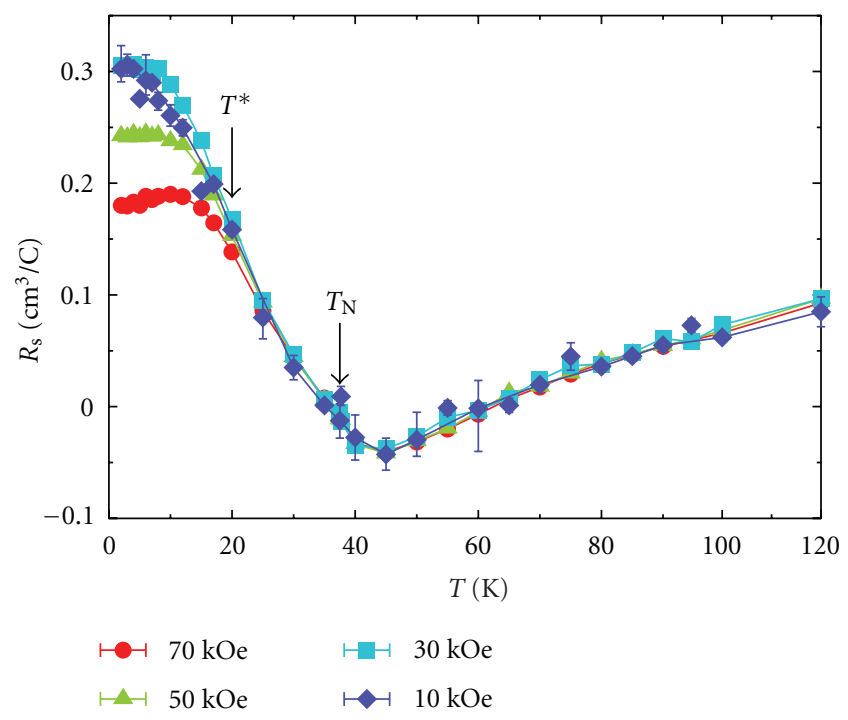

(b)

Figure 12: (a) A plot of $\rho_{x y}$ as a function of $H$ at various temperatures measured using single crystals of $\mathrm{PdCrO}_{2}$. A departure from linear behaviour is seen below $T=30 \mathrm{~K}$, followed by the development of a broad hump between 10-40 kOe. (b) Calculation of the anomalous Hall coefficient, $R_{S}$, using (4) at various fields. A clear field dependency is observed below $T^{*}$. Figures are taken from [16].

the experimental findings from the relevant materials. We have described how the Berry phase mechanism included in the spin orbit interaction-based Karplus and Luttinger theory of collinear ferromagnets has also been adapted for the pyrochlore-based frustrated magnets. However, the exact electronic origin of the Berry phase remains in question. The spin chirality model is unable to consistently reproduce the experimental results, while an orbital-based description remains to be rigorously tested. The discovery of geometrically frustrated materials that display exotic AHE behaviours will provide important opportunities to test theoretical predictions and to determine which mechanisms are important. Furthermore, the large room temperature Hall resistivity of $\mathrm{Fe}_{3} \mathrm{Sn}_{2}$, dominated by the anomalous component, opens the opportunity for this fascinating class of materials to be used in the creation and control of the spinpolarised currents required for spintronics devices.

\section{References}

[1] S. A. Wolf, D. D. Awschalom, R. A. Buhrman et al., "Spintronics: a spin-based electronics vision for the future," Science, vol. 294, no. 5546, pp. 1488-1495, 2001.

[2] D. D. Awschalom and M. E. Flatté, "Challenges for semiconductor spintronics," Nature Physics, vol. 3, no. 3, pp. 153-159, 2007.

[3] Y. Taguchi, Y. Oohara, H. Yoshizawa, N. Nagaosa, and Y. Tokura, "Spin chirality, berry phase, and anomalous hall effect in a frustrated ferromagnet," Science, vol. 291, no. 5513, pp. 2573-2576, 2001.

[4] T. Tomizawa and H. Kontani, "Anomalous Hall effect due to noncollinearity in pyrochlore compounds: role of orbital Aharonov-Bohm effect," Physical Review B, vol. 82, no. 10, Article ID 104412, pp. 104412-1-104412-14, 2010.

[5] Y. Yasui, T. Kageyama, T. Moyoshi, M. Soda, M. Sato, and K. Kakurai, "Studies of anomalous Hall effect and magnetic structure of $\mathrm{Nd}_{2} \mathrm{Mo}_{2} \mathrm{O}_{7}$ - Test of chirality mechanism," Journal of the Physical Society of Japan, vol. 75, no. 8, Article ID 084711, 2006.

[6] Y. Machida, S. Nakatsuji, S. Onoda, T. Tayama, and T. Sakakibara, "Time-reversal symmetry breaking and spontaneous Hall effect without magnetic dipole order," Nature, vol. 463, no. 7278, pp. 210-213, 2010.

[7] R. Karplus and J. M. Luttinger, "Hall effect in ferromagnetics," Physical Review, vol. 95, no. 5, pp. 1154-1160, 1954.

[8] Z. Fang, N. Nagaosa, K. S. Takahashi et al., "The anomalous Hall effect and magnetic monopoles in momentum space," Science, vol. 302, no. 5642, pp. 92-95, 2003.

[9] Y. Machida, S. Nakatsuji, Y. Maeno, T. Tayama, T. Sakakibara, and S. Onoda, "Unconventional anomalous hall effect enhanced by a noncoplanar spin texture in the frustrated kondo lattice $\mathrm{Pr}_{2} \mathrm{Ir}_{2} \mathrm{O}_{7}$," Physical Review Letters, vol. 98, no. 5, Article ID 057203, 2007.

[10] M. V. Berry, "Quantal phase factors accompanying adiabatic changes," Proceedings of the Royal Society A, vol. 392, no. 1802, pp. 45-57, 1984.

[11] S. Iguchi, N. Hanasaki, and Y. Tokura, "Scaling of anomalous hall resistivity in $\mathrm{Nd}_{2}\left(\mathrm{Mo}_{1-x} \mathrm{Nb}_{x}\right)_{2} \mathrm{O}_{7}$ with spin chirality," Physical Review Letters, vol. 99, no. 7, Article ID 077202, 2007.

[12] L. Balicas, S. Nakatsuji, Y. Machida, and S. Onoda, "Anisotropic hysteretic Hall effect and magnetic control of chiral domains in the chiral spin states of $\operatorname{Pr}_{2} \operatorname{Ir}_{2} \mathrm{O}_{7}$, " Physical Review Letters, vol. 106, no. 21, Article ID 217204, 2011.

[13] L. A. Fenner, A. A. Dee, and A. S. Wills, "Non-collinearity and spin frustration in the itinerant kagome ferromagnet $\mathrm{Fe}_{3} \mathrm{Sn}_{2}$," Journal of Physics Condensed Matter, vol. 21, no. 45, Article ID 452202, 2009.

[14] T. Kida, L. A. Fenner, A. A. Dee et al., "The giant anomalous Hall effect in the ferromagnet $\mathrm{Fe}_{3} \mathrm{Sn}_{2}$-a frustrated kagome metal," Journal of Physics, vol. 23, no. 11, Article ID 112205, 2011.

[15] H. Takatsu, H. Yoshizawa, S. Yonezawa, and Y. Maeno, "Critical behavior of the metallic triangular-lattice Heisenberg 
antiferromagnet $\mathrm{PdCrO}_{2}$," Physical Review B, vol. 79, no. 10, Article ID 104424, 2009.

[16] H. Takatsu, S. Yonezawa, S. Fujimoto, and Y. Maeno, "Unconventional anomalous hall effect in the metallic triangularlattice magnet $\mathrm{PdCrO}_{2}$," Physical Review Letters, vol. 105, no. 13, Article ID 137201, 2010.

[17] Y. Aharonov and D. Bohm, "Significance of electromagnetic potentials in the quantum theory," Physical Review, vol. 115, no. 3, pp. 485-491, 1959.

[18] Y. Maeno, H. Hashimoto, K. Yoshida et al., "Superconductivity in a layered perovskite without copper," Nature, vol. 372, no. 6506, pp. 532-534, 1994.

[19] J. M. Longo, P. M. Raccah, and J. B. Goodenough, "Magnetic properties of $\mathrm{SrRuO}_{3}$ and $\mathrm{CaRuO}_{3}$," Journal of Applied Physics, vol. 39, no. 2, pp. 1327-1328, 1968.

[20] C. W. Jones, P. D. Battle, P. Lightfoot et al., "The structure of $\mathrm{SrRuO}_{3}$ by time-of-flight neutron powder diffraction," Acta Crystallographica C, vol. 45, pp. 365-367, 1989.

[21] J. S. Gardner, G. Balakrishnan, and D. M. Paul, "Neutron powder diffraction studies of $\mathrm{Sr}_{2} \mathrm{RuO}_{4}$ and $\mathrm{SrRuO}_{3}$," Physica C, vol. 252, no. 3-4, pp. 303-307, 1995.

[22] S. N. Bushmeleva, V. Y. Pomjakushin, E. V. Pomjakushina, D. V. Sheptyakov, and A. M. Balagurov, "Evidence for the band ferromagnetism in $\mathrm{SrRuO}_{3}$ from neutron diffraction," Journal of Magnetism and Magnetic Materials, vol. 305, no. 2, pp. 491496, 2006.

[23] T. Kiyama, K. Yoshimura, K. Kosuge, Y. Ikeda, and Y. Bando, "Invar effect of $\mathrm{SrRuO}_{3}$ : itinerant electron magnetism of $\mathrm{Ru}$ 4d electrons," Physical Review B, vol. 54, no. 2, pp. R756-R759, 1996.

[24] D. J. Singh, "Electronic and magnetic properties of the $4 \mathrm{~d}$ itinerant ferromagnet $\mathrm{SrRuO}_{3}$," Journal of Applied Physics, vol. 79, no. 8, pp. 4818-4820, 1996.

[25] P. B. Allen, H. Berger, O. Chauvet et al., "Transport properties, thermodynamic properties, and electronic structure of $\mathrm{SrRuO}_{3}$," Physical Review B, vol. 53, no. 8, pp. 4393-4398, 1996.

[26] R. Mathieu, A. Asamitsu, H. Yamada et al., "Scaling of the anomalous hall effect in $\mathrm{Sr}_{1-x} \mathrm{Ca}_{x} \mathrm{RuO}_{3}$," Physical Review Letters, vol. 93, no. 1, Article ID 016602, 2004.

[27] M. Sato, "Unusual behavior of the anomalous Hall effect of systems with non-trivial magnetic structures," Journal of Magnetism and Magnetic Materials, vol. 310, no. 2, pp. 10211023, 2007.

[28] T. Tomizawa and H. Kontani, "Anomalous Hall effect in the $\mathrm{t}_{2 g}$ orbital kagome lattice due to noncollinearity: significance of the orbital Aharonov-Bohm effect," Physical Review B, vol. 80, no. 10, Article ID 100401, 2009.

[29] S. Xu, Y. Moritomo, K. Oikawa, T. Kamiyama, and A. Nakamura, "Lattice structural change at ferromagnetic transition in $\mathrm{Nd}_{2} \mathrm{Mo}_{2} \mathrm{O}_{7}$," Journal of the Physical Society of Japan, vol. 70, no. 8, pp. 2239-2241, 2001.

[30] S. Onoda and N. Nagaosa, "Spin chirality fluctuations and anomalous Hall effect in itinerant ferromagnets," Physical Review Letters, vol. 90, no. 19, Article ID 196602, 2003.

[31] D. Yanagishima and Y. Maeno, "Metal-nonmetal changeover in pyrochlore iridates," Journal of the Physical Society of Japan, vol. 70, no. 10, pp. 2880-2883, 2001.

[32] S. Nakatsuji, Y. Machida, Y. Maeno et al., "Metallic spinliquid behavior of the geometrically frustrated kondo lattice $\mathrm{Pr}_{2} \mathrm{Ir}_{2} \mathrm{O}_{7}$," Physical Review Letters, vol. 96, no. 8, Article ID 087204, 2006.

[33] D. E. MacLaughlin, Y. Ohta, Y. Machida et al., "Weak quasistatic magnetism in the frustrated Kondo lattice $\mathrm{Pr}_{2} \mathrm{Ir}_{2} \mathrm{O}_{7}$," Physica B, vol. 404, no. 5-7, pp. 667-670, 2009.
[34] G. Le Caer, B. Malaman, and B. Roques, "Mossbauer effect study of $\mathrm{Fe}_{3} \mathrm{Sn}_{2}$," Journal of Physics F, vol. 8, no. 2, pp. 323336, 1978.

[35] K. S. Heritage, B. Bryant, Y.-A. Soh et al., Unpublishedwork, 2011.

[36] R. D. Shannon, D. B. Rogers, and C. T. Prewitt, "Chemistry of noble metal oxides. I. Syntheses and properties of $\mathrm{ABO} 2$ delafossite compounds," Inorganic Chemistry, vol. 10, no. 4, pp. 713-718, 1971.

[37] J.-P. Doumerc, A. Wichainchai, A. Ammar, M. Pouchard, and P. Hagenmuller, "On magnetic properties of some oxides with delafossite-type structure," Materials Research Bulletin, vol. 21, no. 6, pp. 745-752, 1986. 

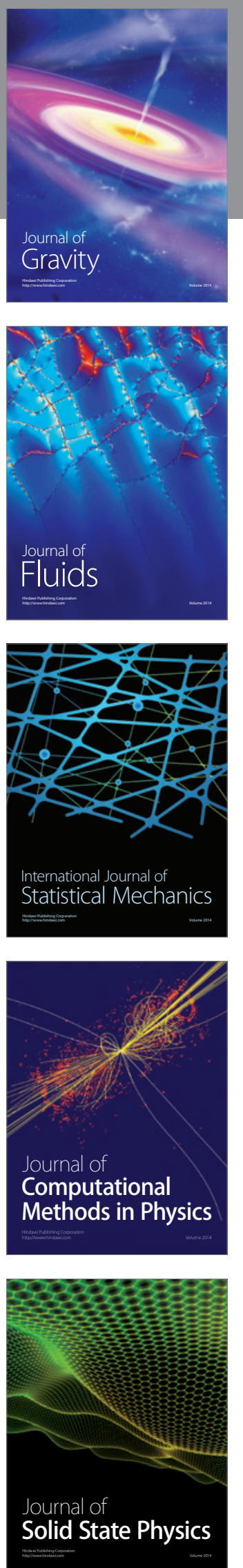

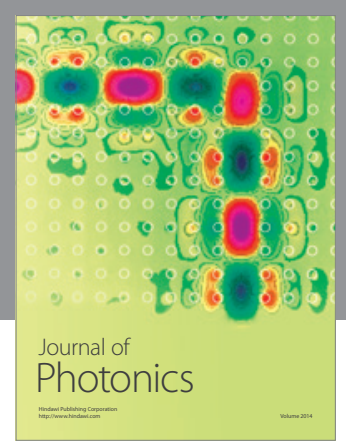

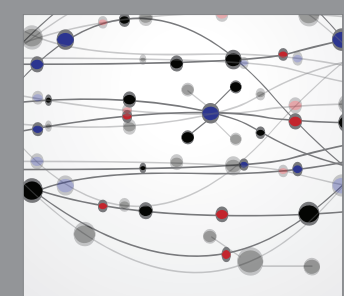

The Scientific World Journal
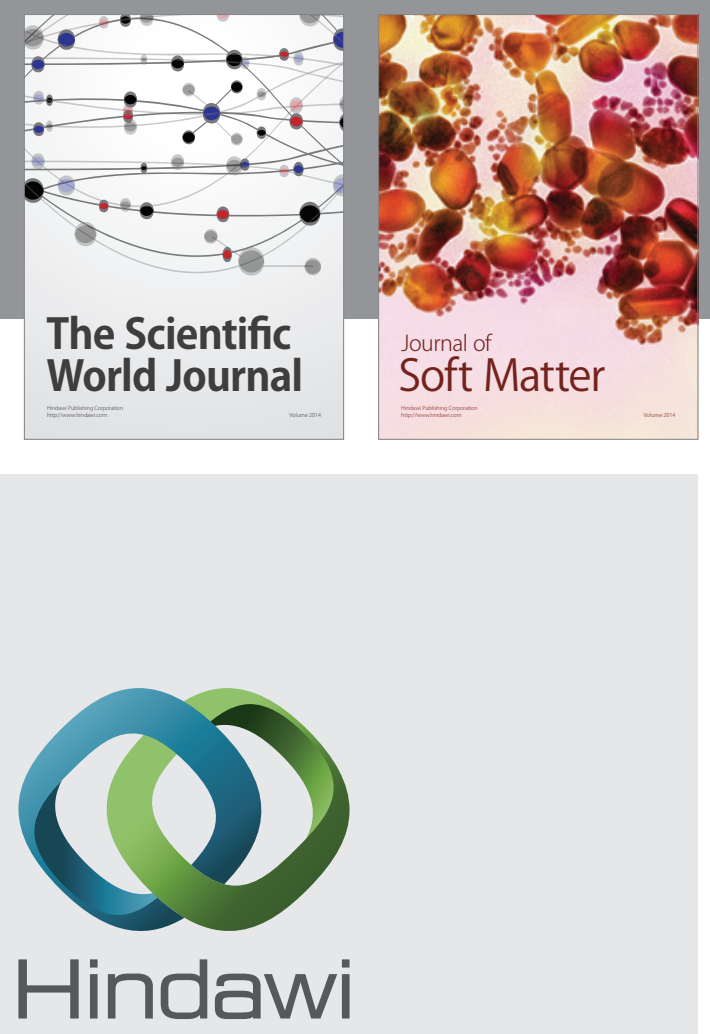

Submit your manuscripts at

http://www.hindawi.com
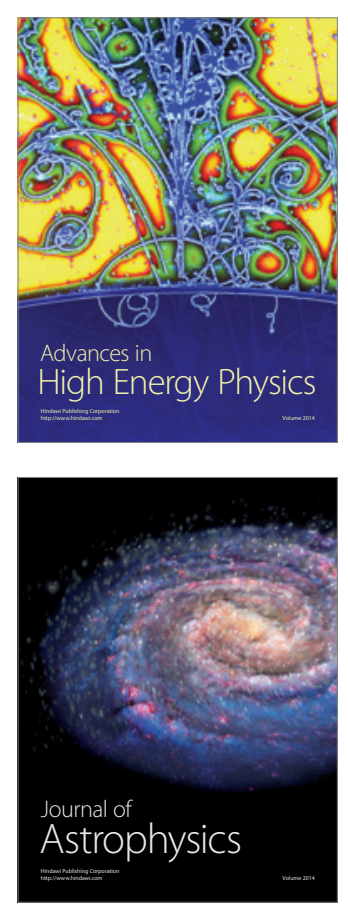
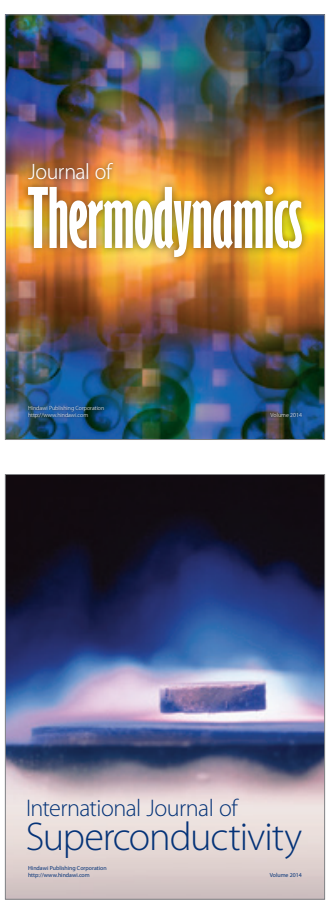
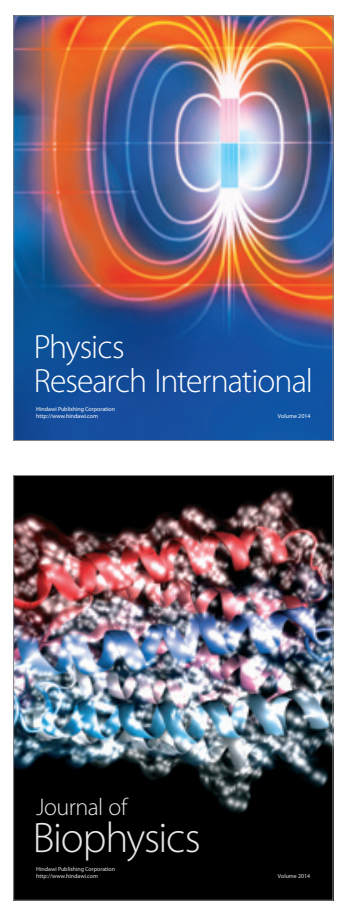
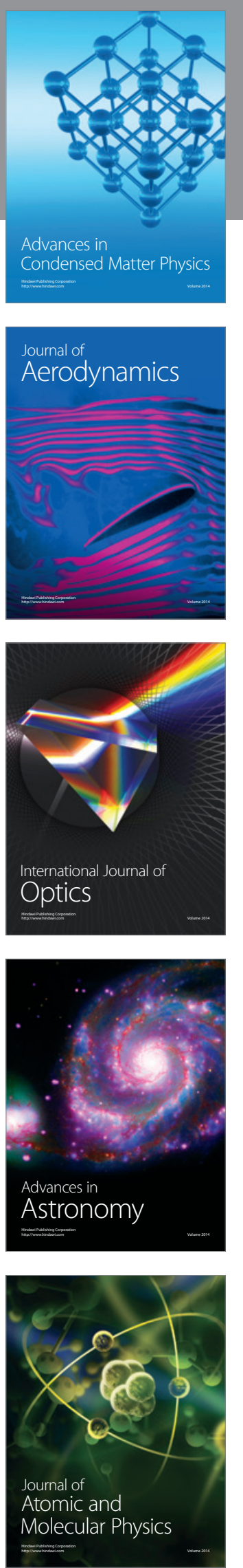